\title{
Collisions, added mass, speed increase, and energy transfer via quantum entanglement
}

\author{
Boris Lagutin
}

Funding: The author(s) received no specific funding for this work.

Potential competing interests: The author(s) declared that no potential competing interests exist.

\section{Abstract}

This paper (preprint) is the author's analytical research of elastic and inelastic collisions (impacts) of two bodies in a linear motion. An experiment (inelastic collision) described in the paper is designed to understand how an additional mass can physically (not in physical formulas) make a final speed increase of one of two colliding bodies. A first body (big block), which is linearly moving at some constant speed, hits a second body (small block) which is motionless. Accelerations of and final speeds of the blocks, and the impact time are measured. Then, some additional mass is assembled to the big block so that a length of the big block can stay the same. Next, the big block (with an added mass) hits the small block keeping the initial speeds of the blocks the same. Accelerations of and final speeds of the blocks, and the impact time are measured. In according to the classical mechanics formulas, a final speed of the small block gets higher if a total mass of the big block is increased, even though the initial speeds of the blocks do not change. However, it is unclear how a mass, which is added to the big block, may physically make an increase of the small block final speed if the initial speeds of and lengths of the colliding blocks do not change. A speed of shock waves is the same in the two blocks if they are made of the same material. Based on the physics of collision, if a length of the small block is less than a length of the big block, then the small block must begin to move when its shock wave returns to the impact area before a shock wave in the big block returns to the impact area. In other words, the small block must begin to move at the same time in the collision with or without an added mass, so the initial speeds of and lengths of the two blocks do not change. Therefore, the physical impact time must be the same for the collision with or without an added mass. Hence, the blocks cannot squeeze each other faster or longer during the physical impact when an added mass is assembled to the big block. In such conditions, the final speed increase of the small block can physically happen in the way that the small block either gets a higher acceleration or accelerates for a longer time after the physical impact between the blocks (with an added mass) ends. The current physics does not explain how physically (not in the formulas) an added mass can make the small block accelerate faster or longer after the physical impact (contact) ends. Therefore, the current collision physics appears not to explain the collision process completely when the small block final speed becomes higher despite the same impact time. The author assumes that maybe the interactions between the particles in the experimental collision may be based on some 
sort of quantum entanglement, anisotropy, relativity, that is, without any fields, or the energy transfer between fields happens via some sort of quantum entanglement anisotropically. Though, such an assumption requires a supplementary experimental research.

Introduction

Elastic and inelastic collisions of two bodies (solid blocks) are analyzed in this paper. To better understand what is going on during elastic and inelastic collisions, let's first analyze an elastic collision of two identical particles.

Assume there are two identical particles (charges) which have the same masses and fields (field lines are depicted) [1], see Figure \#1.

\section{Before the collision}
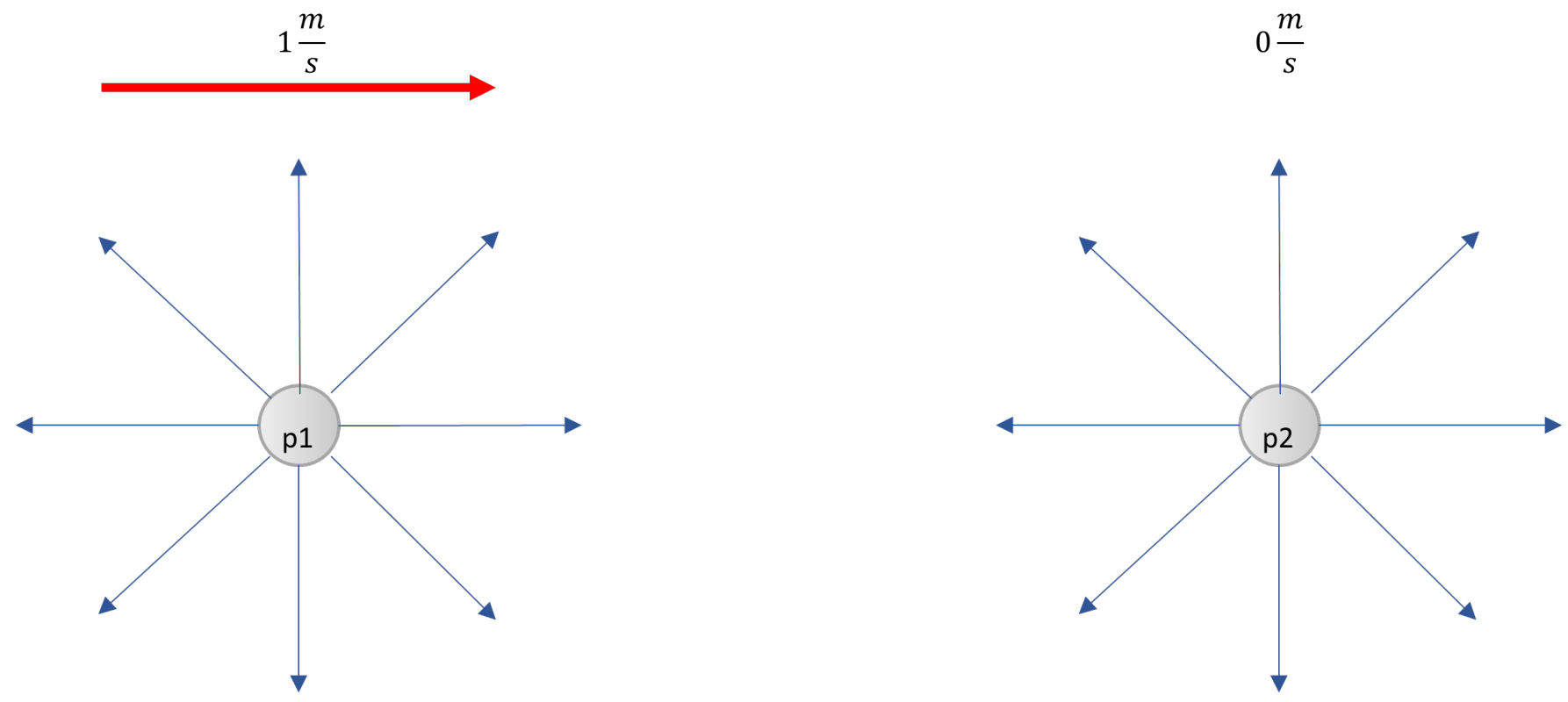

Assume that a particle $p 2$ is not exerted by any forces before the collision and is motionless. A particle $p 1$ reaches $p 2$ at a uniform speed 1 meter per second. At some point, the field of $p 1$ begins to compress the field of $p 2$ [2] so that the initial speeds of both particles begin to change: the $p 1$ speed decreases and the p2 speed increases. As a result, the fields of both particles are compressed at some distance $x$, see Figure \#2. 


\section{The collision starts}

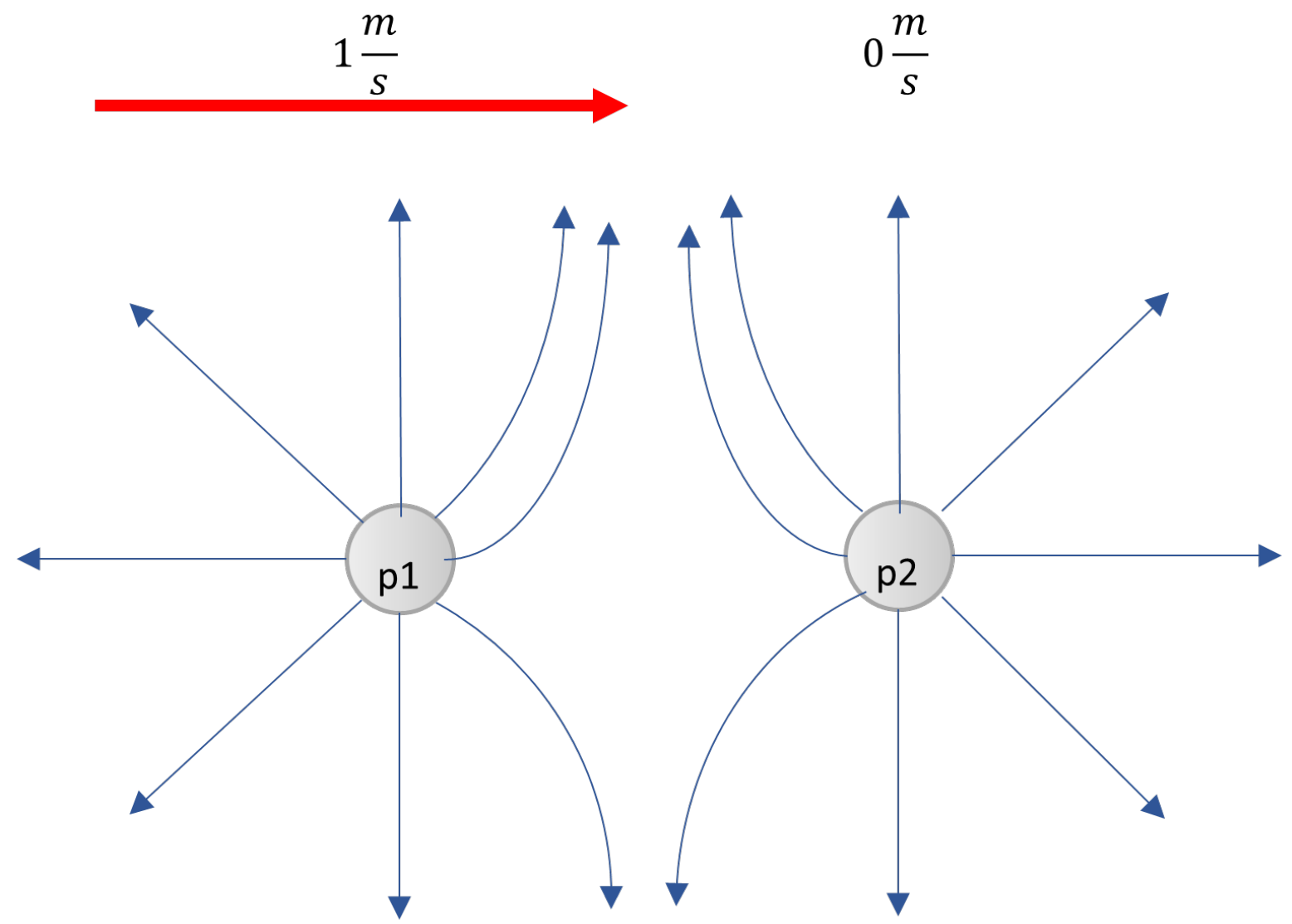

To sum up, the interactions of the particles happen by their fields. Notice that the centers of mass of the particles are along one line.

Now, let's consider a more complicated process when two different groups [3] of identical particles of steel collide with one another along one axis (uniaxially [4]). The fields of all the particles are identical too. Assume there are not any friction, air resistance, other forces exerted on the groups. The left group of the particles is bigger than the right group of the particles. A length $\boldsymbol{L}_{\mathbf{1}}$ of the left group is more than a length $\boldsymbol{L}_{\mathbf{2}}$ of the right group. The left group begins to move toward the right group at a uniform speed 1 meter per second at time $\boldsymbol{t}=\mathbf{0}$, see Figure \#3. 


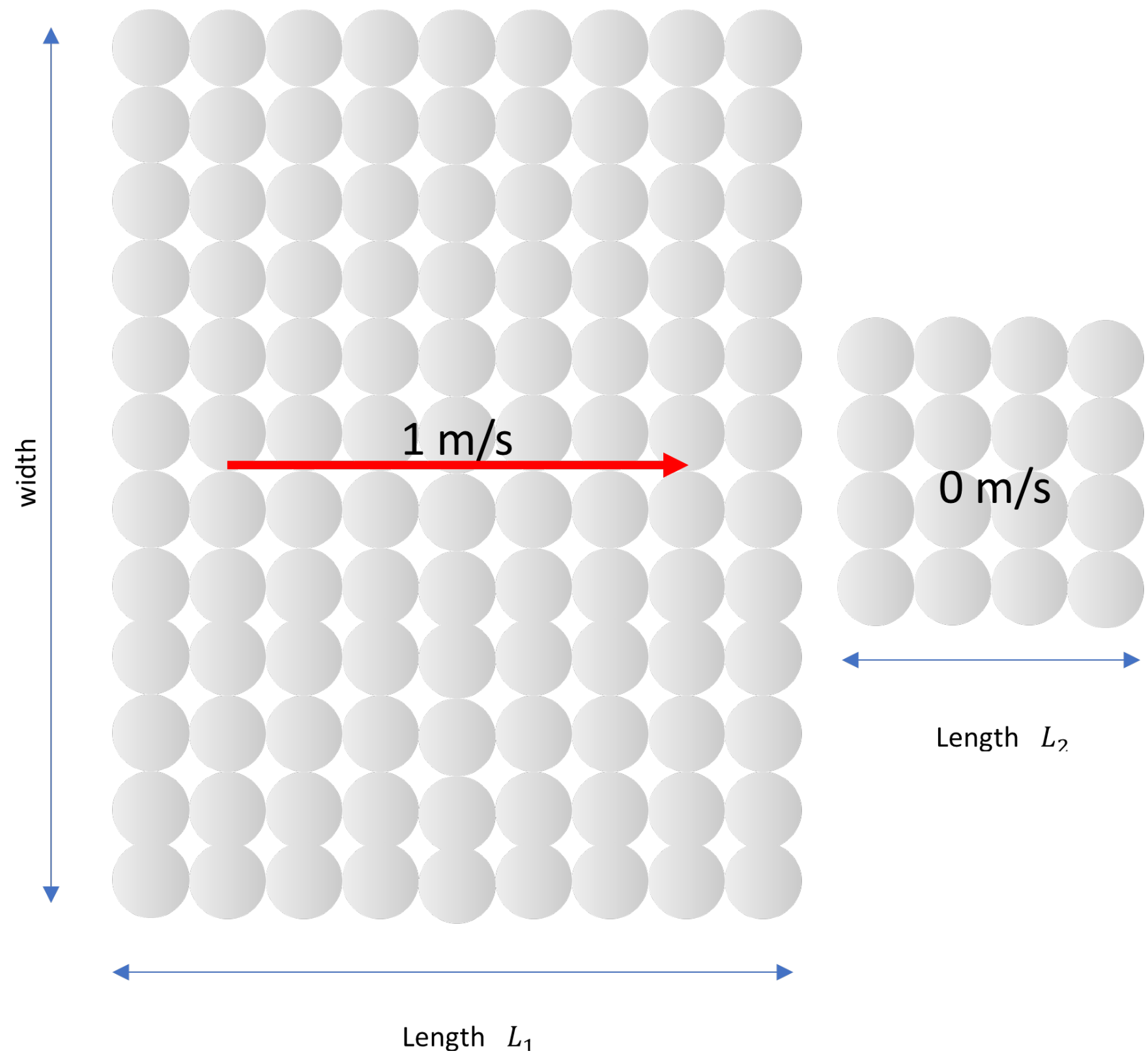

When the collision (impact) starts, the fields of the left group surface particles begin to compress the fields of the right group surface particles along one axis at the left group initial speed, see Figure \#4. 


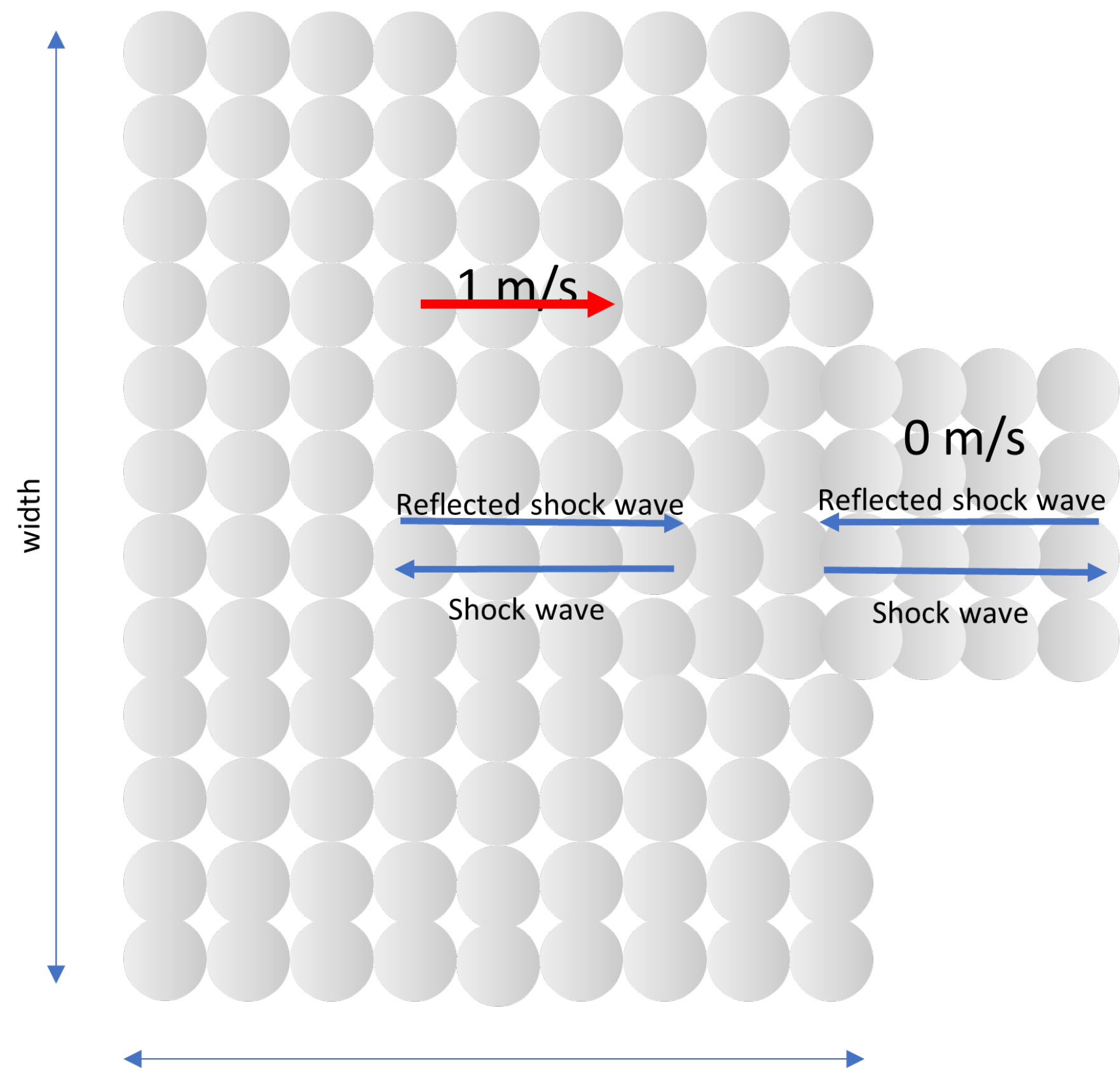

Length $L_{1}$

Assume that the collision between the groups starts at time $\boldsymbol{t}_{\mathbf{1}}$. The shock wave propagates at the same speed in both groups because they represent the same material (steel) [5]. The impact should end when the shock waves return to the impact area [6, 7]. However, the shock wave reflected from the right end of the right group returns to the impact area earlier than the reflected shock wave in the left group returns from its left end because $\boldsymbol{L}_{\mathbf{2}}$ is less than $\boldsymbol{L}_{\mathbf{1}}$. Hence, the impact should end when the shock wave in the right group returns to the impact area. The groups both begin to deform at the impact area along one axis. The deformation is assumed to be elastic, that is, the groups restore their initial shapes without strains after the impact $[5,6,7]$.

The linear momentum of any mass is defined by a mass $\boldsymbol{m}$ multiplied by its velocity $\boldsymbol{v}$ [8]:

$$
n=m i(1)
$$


Next, increase a width of the left group but keep the same length so that the centers of the mass of the groups are in line, see Figure \#5.

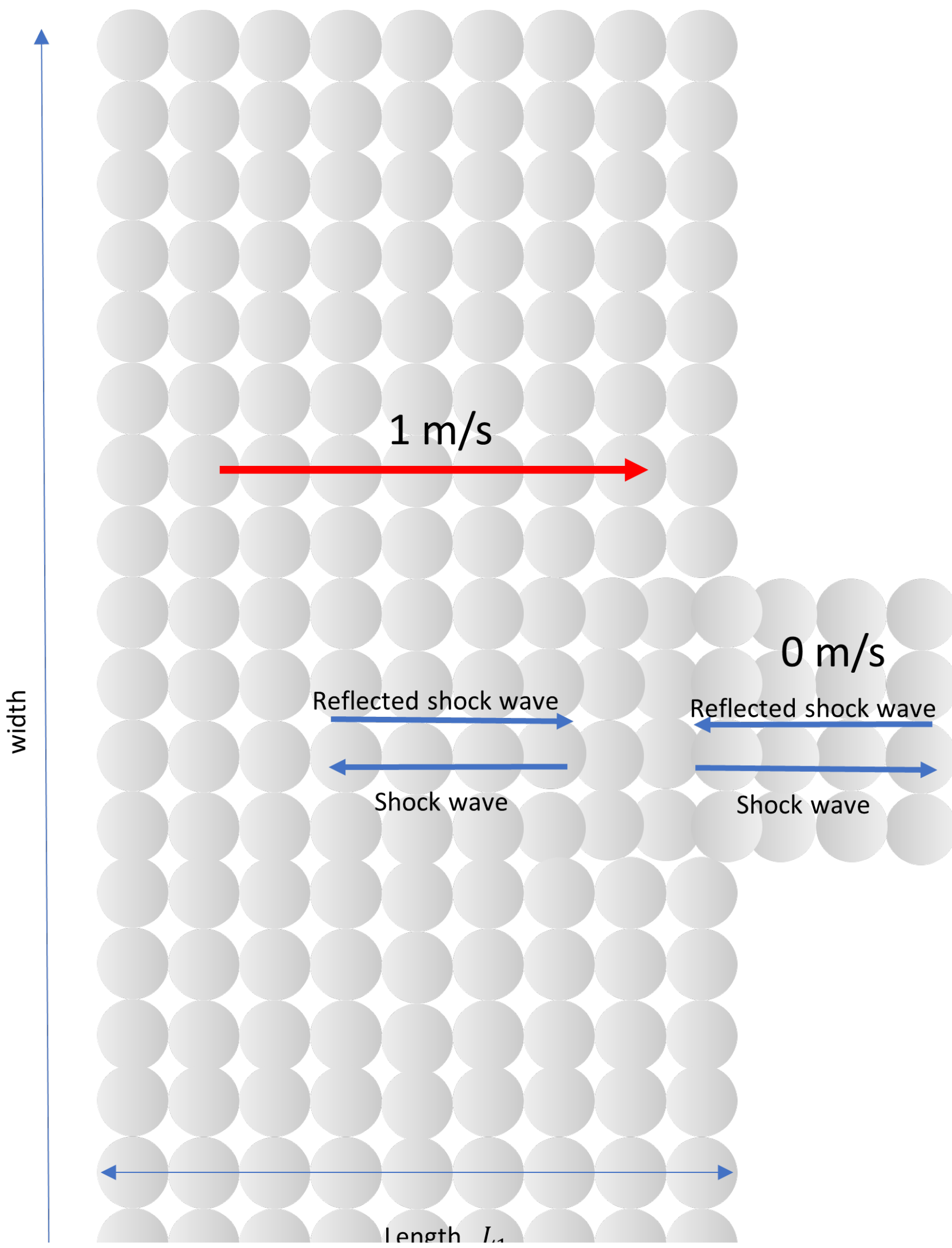




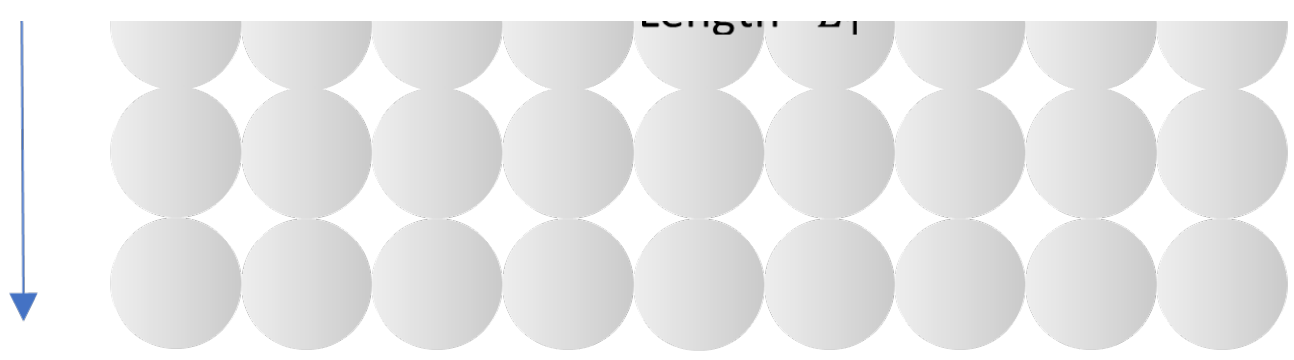

Therefore, a total mass of the left group becomes larger but a mass of the right group stays unchanged. The left group hits the right group at the same initial speed. Likely the deformation in the collision (impact) before increasing the left group, the deformation of the groups happens along one axis (uniaxially).

Because a total mass of the left group increases, a force exerted on each group becomes larger than that force in the collision before increasing the left group. The force $\boldsymbol{F}$ (magnitude of the force) is calculated based on the following formula via impulse:

$$
F=\left(m_{\text {group }} v_{\text {group }}-m_{\text {group }} i_{\text {group }}\right) / t_{\text {collision }}
$$

where $\boldsymbol{m}_{\text {group }}$ is a total mass of one of the groups, $\boldsymbol{v}_{\text {group }}$ is a final speed (after collision) of a group, $\boldsymbol{i}_{\text {group }}$ is an initial speed (before collision) of a group, $\boldsymbol{t}_{\text {collision }}$ is the collision time [9]. The same force $\boldsymbol{F}$ is exerted by the groups on each other in according to Newton's third law [10]. The deformation delta $\boldsymbol{L}$ of each group should be larger after increasing the left group because the force $\boldsymbol{F}$ becomes larger based on the following equation:

$$
\Delta L=(F L) /(A E),(3)
$$

where $\boldsymbol{L}$ is a length of a group before the deformation, $\boldsymbol{A}$ is the collision contact area between the groups (impact area), $\boldsymbol{E}$ is Young's modulus (elastic modulus) for steel. The equation (3) can be derived from the formulas for epsilon (engineering strain), sigma (applied or engineering stress), and $\boldsymbol{E}$ (Young's modulus or elastic modulus) [11]. The length $\boldsymbol{L}$ of each group and the impact area $\boldsymbol{A}$ do not change in the collisions. Notice that the deformation of the left group is always less than the deformation of the right group if $\mathbf{L}_{\mathbf{2}}$ is less than $\boldsymbol{L}_{\mathbf{1}}$ based on the equation (3). It should be mentioned that the fields of the particles are compressed during the process of the elastic deformation. When the pressure in the contact (impact) area reduces to zero, all the fields of the particles restore their initial state.

The deformation of both groups gets larger because of the larger force $\boldsymbol{F}$ based on the equation (3). If the initial speeds of the groups do not not change, then the larger deformation can physically happen only if the impact time gets longer.

If the impact time increases after the left group gets bigger, it induces the following point. When the collision ends at some time $\boldsymbol{t}_{\mathbf{2}}$ (before the left group being increased), the right group begins to move at some speed $\boldsymbol{v}_{\mathbf{2}}$. Therefore, the right group gets enough kinetic energy to begin to move at $\boldsymbol{t}_{\mathbf{2}}$. After the left group gets increased and if the impact time gets longer, then the right group should begin to move at some time $\boldsymbol{t}_{\mathbf{2}}{ }^{\prime}$. The latter is supposed to be longer than $\boldsymbol{t}_{\mathbf{2}}$. However, it is unclear why the right group should not start to move at $\boldsymbol{t}_{\mathbf{2}}$ if the right group gets enough kinetic energy to begin to move at a 
speed $\boldsymbol{v}_{\mathbf{2}}$ at time $\boldsymbol{t}_{\mathbf{2}}$. Notice that the fields of the particles at the impact area compress each other at the same speed in both collisions and a speed of the shock wave propagation does not change. Moreover, if a length of the right group is less than a length of the left group, then the right group must begin its own movement when the shock wave (in the right group) returns to the impact area. Hence, nothing prevents the right group to begin to move at $\boldsymbol{t}_{\mathbf{2}}$ in the collision with the enlarged left group.

Next, let's first analyze an elastic collision (ideal impact) of two masses that consist of the particles, of course. Assume that there are two different masses (two solid blocks) that are made of steel. These blocks freely move on wheels along a smooth straight metal floor or rails in vacuum (friction is negligible and no air resistance). A big block (Block \#1) is $50 \mathrm{~kg}$ and a small block (Block \#2) is just $1 \mathrm{~kg}$. These two blocks collide with each other in an elastic collision (Situation \#1), see Figure \#6.

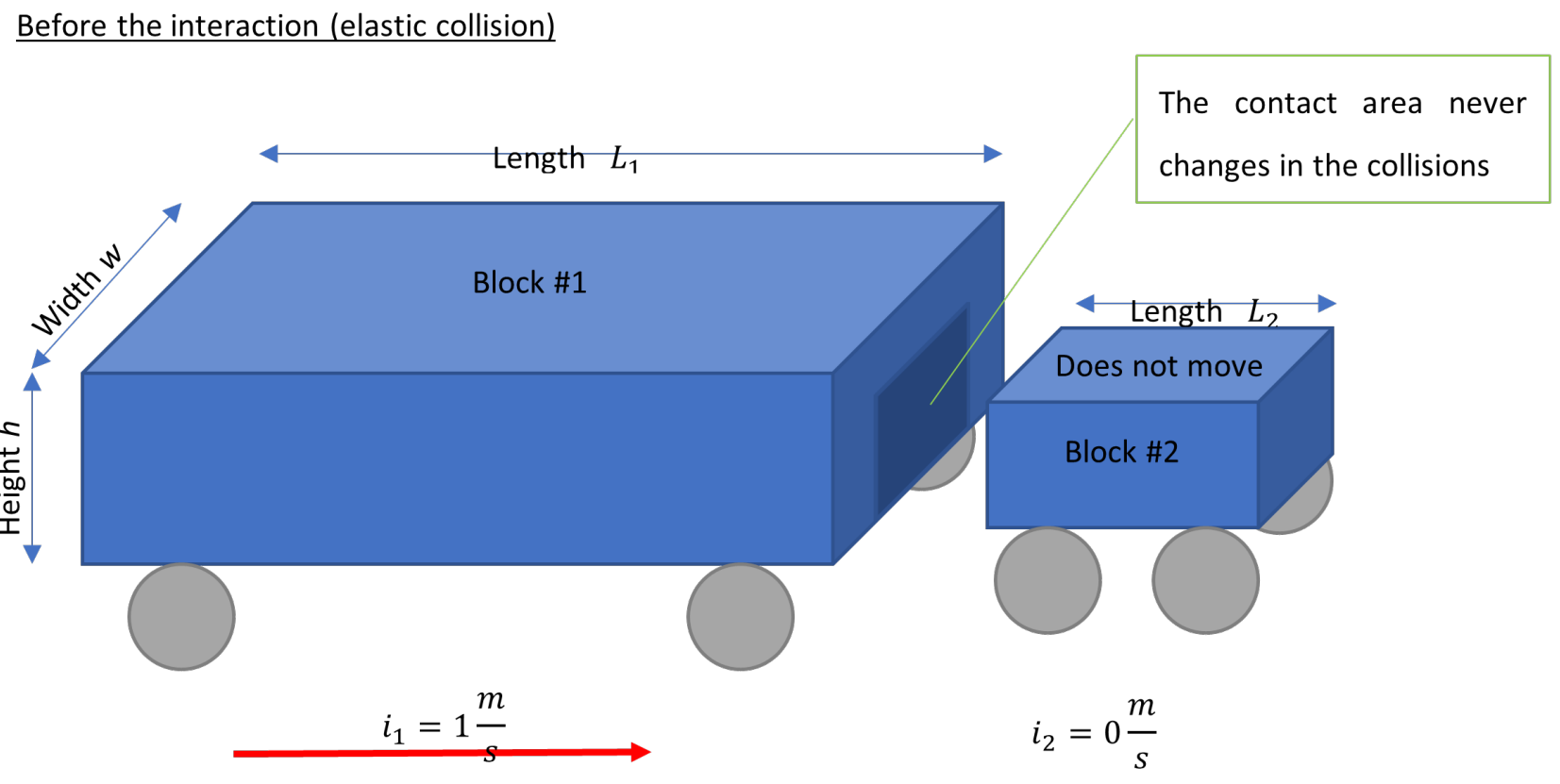

\section{After the interaction (elastic collision)}

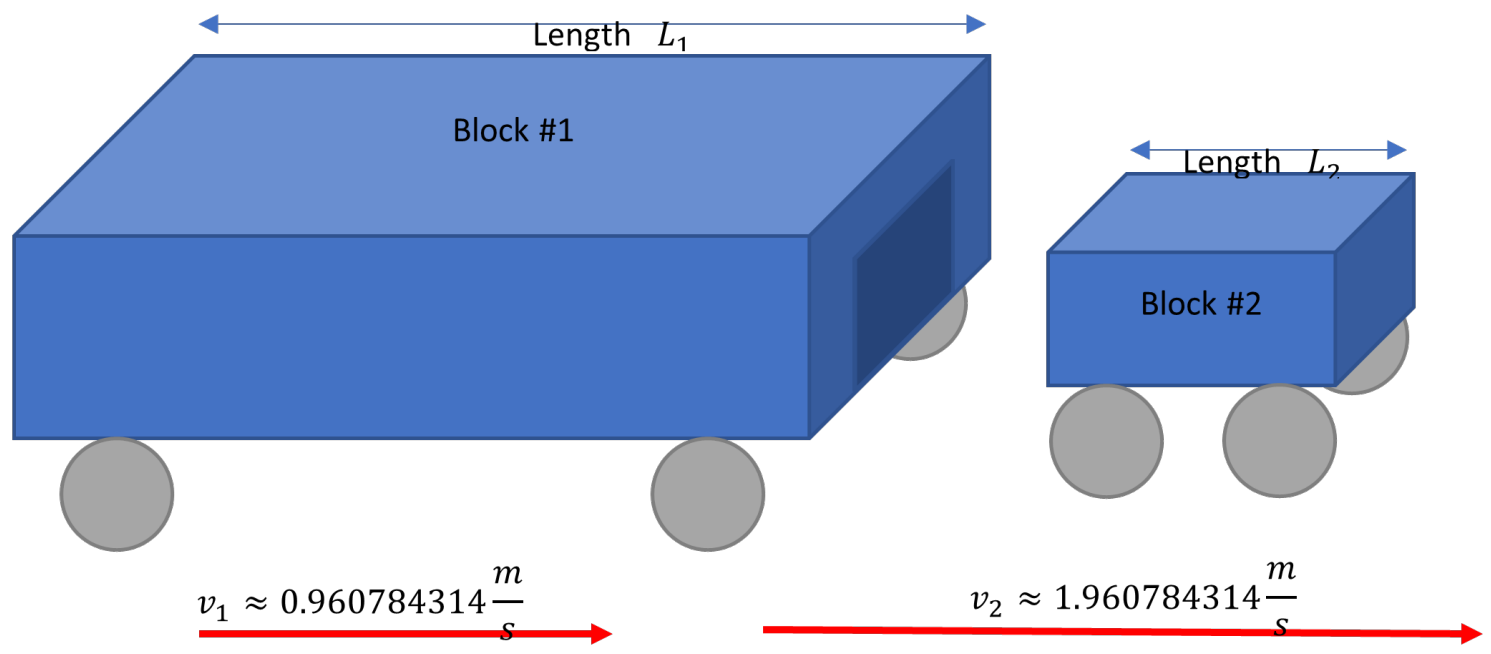

Notice that the centers of mass of each block must be in line during the collision to avoid rotation and 
vibration. The big block initial speed (before the impact) is $\mathbf{1}$ meter per second. The small block initial speed (before the impact) is zero. The elastic collision means that no any kinetic energy is lost, that is, turns into heat, sound waves during the collision [12]. If the momentum of the small block is zero before the impact, then the momentum of the big block before the impact must be equal to a sum of the momenta of the two blocks after the impact. The appropriate formula defines the conservation of the linear momentum when two different bodies (blocks) interact in the elastic collision, that is, when no any kinetic energy is lost in the collision:

$$
m_{1} i_{1}+m_{2} i_{2}=m_{1} v_{1}+m_{2} v_{2}
$$

where $\boldsymbol{m}_{\mathbf{1}}$ is a mass of the big block (Block \#1), $\boldsymbol{i}_{\mathbf{1}}$ is the big block initial speed $\mathbf{1}$ meter per second (before the impact), $\boldsymbol{m}_{\mathbf{2}}$ is a mass of the small block (Block \#2), $\boldsymbol{i}_{\mathbf{2}}$ is the small block initial speed $\mathbf{0}$ meter per second (before the impact), $\boldsymbol{v}_{\mathbf{1}}$ is the big block final speed (after the impact), $\boldsymbol{v}_{\mathbf{2}}$ is the small block final speed (after the impact) [13].

If the small block initial speed $\boldsymbol{i}_{\mathbf{2}}$ is zero, then the equation (4) becomes:

$$
m_{1} i_{1}+m_{2} 0=m_{1} v_{1}+m_{2} v_{2}
$$

hence:

$$
m_{1} i_{1}=m_{1} v_{1}+m_{2} v_{2}
$$

Therefore, the formula for the kinetic energy is [14]:

$$
\left(m_{1} i_{1}^{2}\right) / 2=\left(m_{1} v_{1}^{2}\right) / 2+\left(m_{2} v_{2}^{2}\right) / 2,
$$

hence, the formulas for $\boldsymbol{v}_{\mathbf{1}}$ and $\boldsymbol{v}_{\mathbf{2}}$ are [15]:

$$
\begin{gathered}
v_{1}=\left(\left(m_{1}-m_{2}\right) /\left(m_{1}+m_{2}\right)\right) i_{1}, \quad(8) \\
v_{2}=\left(\left(2 m_{1}\right) /\left(m_{1}+m_{2}\right)\right) i_{1} \cdot(9)
\end{gathered}
$$

Next, figure out the final speeds of the blocks (after the impact) if the big block initial speed is 1 meter per second:

$$
\begin{gathered}
v_{1}=((50 \mathrm{~kg}-1 \mathrm{~kg}) /(50 \mathrm{~kg}+1 \mathrm{~kg})) 1 \mathrm{~m} / \mathrm{s} \approx 0.960784314 \mathrm{~m} / \mathrm{s}, \quad(10) \\
v_{2}=2((50 \mathrm{~kg}) /(50 \mathrm{~kg}+1 \mathrm{~kg})) 1 \mathrm{~m} / \mathrm{s} \approx 1.960784314 \mathrm{~m} / \mathrm{s} .
\end{gathered}
$$

Therefore, the final speed of the small block is almost $\mathbf{1 . 9 6}$ meters per second. This speed is almost two times more than the big block final speed.

Next, consider Situation \#2 when an additional mass $\mathbf{1 5 0}$ kilograms is assembled to the big block (Block \#1), see Figure \#7. 

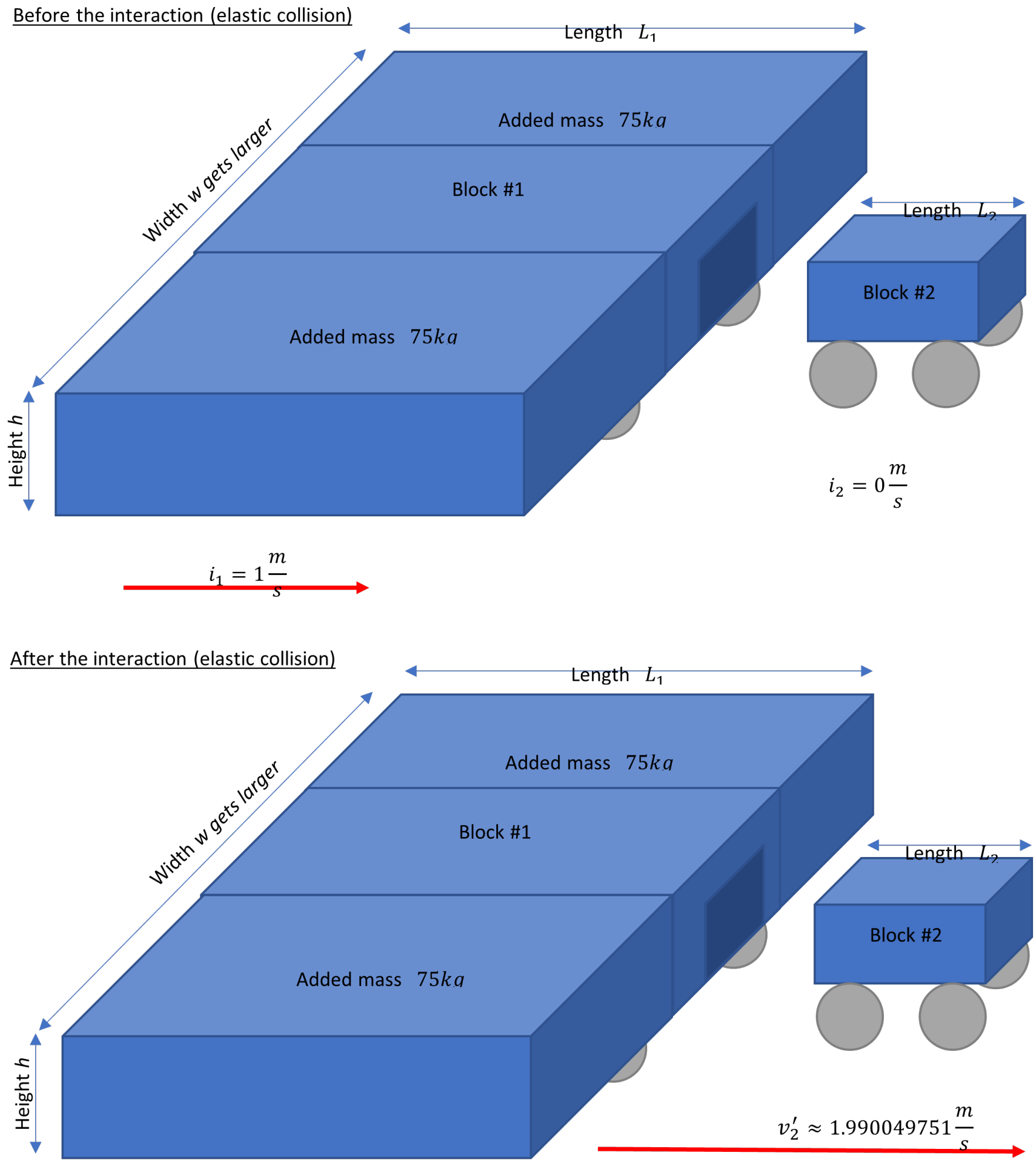

$$
\stackrel{v_{1}^{\prime} \approx 0.990049751 \frac{\mathrm{m}}{\mathrm{s}}}{\longrightarrow}
$$

The additional mass is tightly assembled to Block \#1 so that its length $\boldsymbol{L}_{\mathbf{1}}$ can stay the same. Now, Block \#1 has a total mass of $\mathbf{2 0 0}$ kilograms, but Block \#2 has the same mass of $\mathbf{1}$ kilogram. The initial speed of Block \#1 does not change and is $\mathbf{1}$ meter per second. The initial speed of Block \#2 is zero. Based on the formulas (8) and (9), calculate both a final speed $\mathbf{v}_{\mathbf{1}}$ ' of Block \#1 (with the added mass) and a final speed 
$\boldsymbol{v}_{\mathbf{2}}$ of Block \#2 after the impact with the additional mass:

$$
\begin{aligned}
& v_{1}^{\prime}=((200 \mathrm{~kg}-1 \mathrm{~kg}) /(200 \mathrm{~kg}+1 \mathrm{~kg})) 1 \mathrm{~m} / \mathrm{s} \approx 0.990049751 \mathrm{~m} / \mathrm{s}, \\
& v_{2}=((2 * 200 \mathrm{~kg}) /(200 \mathrm{~kg}+1 \mathrm{~kg})) 1 \mathrm{~m} / \mathrm{s} \approx 1.990049751 \mathrm{~m} / \mathrm{s}
\end{aligned}
$$

Compare the final speed of Block \#2 before the additional mass is assembled to Block \# 1 with the final speed of Block \#2 after the additional mass is assembled to Block \#1:

$$
1.990049751 \mathrm{~m} / \mathrm{s}>1.960784314 \mathrm{~m} / \mathrm{s},(14)
$$

hence:

$$
1.990049751 \mathrm{~m} / \mathrm{s}-1.960784314 \mathrm{~m} / \mathrm{s}=0.029265437 \mathrm{~m} / \mathrm{s} . \quad(15)
$$

It is obvious that Block \#2 increases its final speed by $\mathbf{0 . 0 2 9 2 6 5 4 3 7} \mathrm{m} / \mathrm{s}$ after the additional mass $\mathbf{1 5 0}$ kilograms is assembled to Block \#1 based on the equation (13). It should be underscored that the initial speed of Block \#1 does not change. Notice that it does not matter to where the additional mass is assembled based on the formulas (8) and (9). The additional mass may be tightly assembled wherever: on the top or to the bottom, or behind Block \#1, or other positioning. However, the additional mass must be assembled so that the impact contact area can stay unchanged.

The final speed increase of Block \#2 after adding the additional mass is explained by a larger force exerted by Block \#1 (with the additional mass) on Block \#2 during the impact. This force may be calculated via the impulse based on the formula (2):

$$
F=\left(m_{2} v_{2}-m_{2} i_{2}\right) / t_{\text {elastic }}
$$

where $\boldsymbol{t}_{\text {elastic }}$ is the elastic impact time in seconds. Next, figure out a force $\boldsymbol{F}_{\mathbf{1 , 1 , 2}}$ exerted by Block \#1 on Block \#2 before assembling the additional mass and a force $\boldsymbol{F}_{\mathbf{2 , 1 , 2}}$ exerted by Block \#1 on Block \#2 after assembling the additional mass. First of all, calculate the force $\boldsymbol{F}_{\mathbf{1 , 1 , 2}}$ :

$$
F_{1,1,2}=((1 \mathrm{~kg} 1.960784314 \mathrm{~m} / \mathrm{s})-(1 \mathrm{~kg} 0.0 \mathrm{~m} / \mathrm{s})) / t_{\text {elastic }}=(1.960784314 \mathrm{kgm} / \mathrm{s}) / t_{\text {elastic }}
$$

where $\boldsymbol{t}_{\text {elastic }}$ is the impact time before assembling the additional mass. Second, calculate the force $\boldsymbol{F}_{\mathbf{2 , 1 , 2}}$ :

$$
F_{2,1,2}=((1 \mathrm{~kg} 1.990049751 \mathrm{~m} / \mathrm{s})-(1 \mathrm{~kg} 0.0 \mathrm{~m} / \mathrm{s})) / t_{\text {elastic }}^{\prime}=(1.990049751 \mathrm{kgm} / \mathrm{s}) / t_{\text {elastic }}^{\prime}
$$

where $\boldsymbol{t}_{\text {elastic }}{ }^{\prime}$ is the impact time after assembling the additional mass.

In according to Newton's third law, $\boldsymbol{F}_{\mathbf{1 , 1 , 2}}$ must be equal to a force $\boldsymbol{F}_{\mathbf{1 , 2 , 1}}$ exerted by Block \#2 on Block \#1 before assembling the additional mass, and $\boldsymbol{F}_{\mathbf{2 , 1 , 2}}$ must be equal to a force $\boldsymbol{F}_{\mathbf{2 , 2 , 1}}$ exerted by Block \#2 on Block \#1 after assembling the additional mass. Next step, calculate $\boldsymbol{F}_{\mathbf{1 , 2 , 1}}$ and $\boldsymbol{F}_{\mathbf{2 , 2 , 1}}$ :

$$
F_{1,2,1}=((50 \mathrm{~kg} 1 \mathrm{~m} / \mathrm{s})-(50 \mathrm{~kg} 0.960784314 \mathrm{~m} / \mathrm{s})) / t_{\text {elastic }}=(1.9607843 \mathrm{kgm} / \mathrm{s}) / t_{\text {elastic }}
$$




$$
F_{2,2,1}=((200 \mathrm{~kg} 1 \mathrm{~m} / \mathrm{s})-(200 \mathrm{~kg} 0.990049751 \mathrm{~m} / \mathrm{s})) / t_{\text {elastic }}=\left(1.990049751 \mathrm{kgm} / \mathrm{s}^{\prime}\right) / t_{\text {elastic }} .
$$

Let's first compare $\boldsymbol{F}_{\mathbf{1 , 1 , 2}}$ with $\boldsymbol{F}_{\mathbf{1 , 2 , 1}}$ which are forces exerted by the blocks on each other in the collision before the additional mass is assembled to Block \#1:

$$
(1.960784314 \mathrm{kgm} / \mathrm{s}) / t_{\text {elastic }} \cong(1.9607843 \mathrm{kgm} / \mathrm{s}) / t_{\text {elastic }}
$$

then compare $\boldsymbol{F}_{\mathbf{2 , 1 , 2}}$ with $\boldsymbol{F}_{\mathbf{2 , 2}, \mathbf{1}}$ which are forces exerted by the blocks on each other in the collision after the additional mass is assembled to Block \#1:

$$
(1.990049751 \mathrm{kgm} / \mathrm{s}) / t_{\text {elastic }} \cong(1.990049751 \mathrm{kgm} / \mathrm{s}) / t_{\text {elastic }} .
$$

Notice that the difference between $\mathbf{v}_{\mathbf{1}}$ and $\mathbf{v}_{\mathbf{2}}$ or $\mathbf{v}_{\mathbf{1}}{ }^{\prime}$ and $\boldsymbol{v}_{\mathbf{2}}{ }^{\prime}$ is always 1 meter per second:

$$
\begin{aligned}
& 1.960784314 \mathrm{~m} / \mathrm{s}-0.960784314 \mathrm{~m} / \mathrm{s}=1 \mathrm{~m} / \mathrm{s}, \\
& 1.990049751 \mathrm{~m} / \mathrm{s}-0.990049751 \mathrm{~m} / \mathrm{s}=1 \mathrm{~m} / \mathrm{s} .
\end{aligned}
$$

It means that a distance between the blocks grows at the same rate that is $\mathbf{1}$ meter per second after the elastic impact in both situations. That is, the blocks separate from each other at the same distance per a unit of time in Situation \#1 and Situation \#2 after the blocks gain their final speeds. However, it is necessary to analyze an inelastic collision of the blocks to estimate physical conditions properly.

Let's consider a real experiment for Situation \#1 and Situation \#2 which are inelastic collisions happening in the real world. A length of Block \#2 must be less than a length of Block \#1 for the experimental goal. The blocks are made of good quality steel. The blocks must be without external and internal defects. The deformations of the blocks are supposed to be elastic at the experimental speeds. The elastic modulus $\boldsymbol{E}$ for steel is $200 \mathrm{GPa}$ [16]. The initial speed of Block \#1 does not change and is $\mathbf{1}$ meter per second for Situation \#1 and Situation \#2. The initial speed of Block \#2 is zero for Situation \# 1 and Situation \#2. The blocks may be collided with each other in vacuum in both situations in order to reduce air resistance. Practically, friction in the block wheels bearings, friction between the wheels and the floor or rails may be insignificant for calculating the experimental results. To measure the impact time, the blocks are connected to a timer. When the blocks contact each other during the impact, it closes the timer circuit (steel is electrically conductive). When the blocks separate, the timer circuit is open again. Another timer is used to measure a total time of the experiment. The first timer and the second timer must be synchronized. To measure the final speeds and accelerations of the blocks, a video camera is used. Special marks are painted on the blocks. There are many applications which may be used to calculate speeds of bodies based on a video by using the marks. Though, the inelastic (permanent) deformation of the blocks should not happen at low initial speeds of Block \#1, the sizes of the blocks are checked after every impact with an error at least + or -0.01 millimeter.

During the inelastic collisions, some kinetic energy turns into the shock wave (elastic deformation) and heat. It means that the equation (7) changes, that is, the kinetic energy of Block \#1 before the impact of the blocks should be more than the total kinetic energy of the blocks after the impact. Moreover, the final 
speeds of the blocks should change as well. The final speeds should become a bit slower than they are in the elastic collisions. Hence, a final speed of Block \#1 becomes $\boldsymbol{v}_{\mathbf{1} \text { s }}$ and a final speed of Block \#2 becomes $\boldsymbol{v}_{\mathbf{2} \boldsymbol{s}}$. Then, the kinetic energy inequality is:

$$
m_{1} i_{1}^{2} / 2>m_{1} v_{1 s}^{2} / 2+m_{2} v_{2 s}^{2} / 2
$$

therefore, the momentum of Block \#1 before the impact should be more than a sum of the momenta of the two blocks after the impact:

$$
m_{1} i_{1}>m_{1} v_{1 s}+m_{2} v_{2 s} \cdot(26)
$$

Though, it is necessary to remember that a total momentum of the system is conserved. To calculate the final speeds of the blocks in the inelastic collisions, the coefficient of restitution $\boldsymbol{C}_{\boldsymbol{R}}$ is used [17, 18]:

$$
C_{R}=\left(v_{2 s}-v_{1 s}\right) /\left(i_{1}-i_{2}\right) .
$$

Therefore, a final speed of Block \#1 is calculated based on this formula:

$$
v_{1 s}=\left(m_{1} i_{1}+m_{2} i_{2}+m_{2} C_{R}\left(i_{2}-i_{1}\right)\right) /\left(m_{1}+m_{2}\right),
$$

and a final speed of Block \#2 after the inelastic collision is [18, 19]:

$$
v_{2 s}=\left(m_{1} i_{1}+m_{2} i_{2}+m_{1} C_{R}\left(i_{1}-i_{2}\right)\right) /\left(m_{1}+m_{2}\right) .
$$

Consequently, if $\boldsymbol{C}_{\boldsymbol{R}}$ is known before making the experiment (with and without the additional mass), the final speeds of the blocks are defined based on the formulas (28) and (29).

Next, first analyze the inelastic collision in Situation \#1 (without the additional mass). Block \#1 (50 $\mathrm{kg}$ ) collides with Block \#2 $(1 \mathrm{~kg})$. The impact starts at time $\boldsymbol{t}_{\mathbf{1}}$ and ends at time $\boldsymbol{t}_{\mathbf{2}}$ when Block \#2 begins to move at its final speed $\boldsymbol{v}_{\mathbf{2 s}}$, see Figure \#8. 


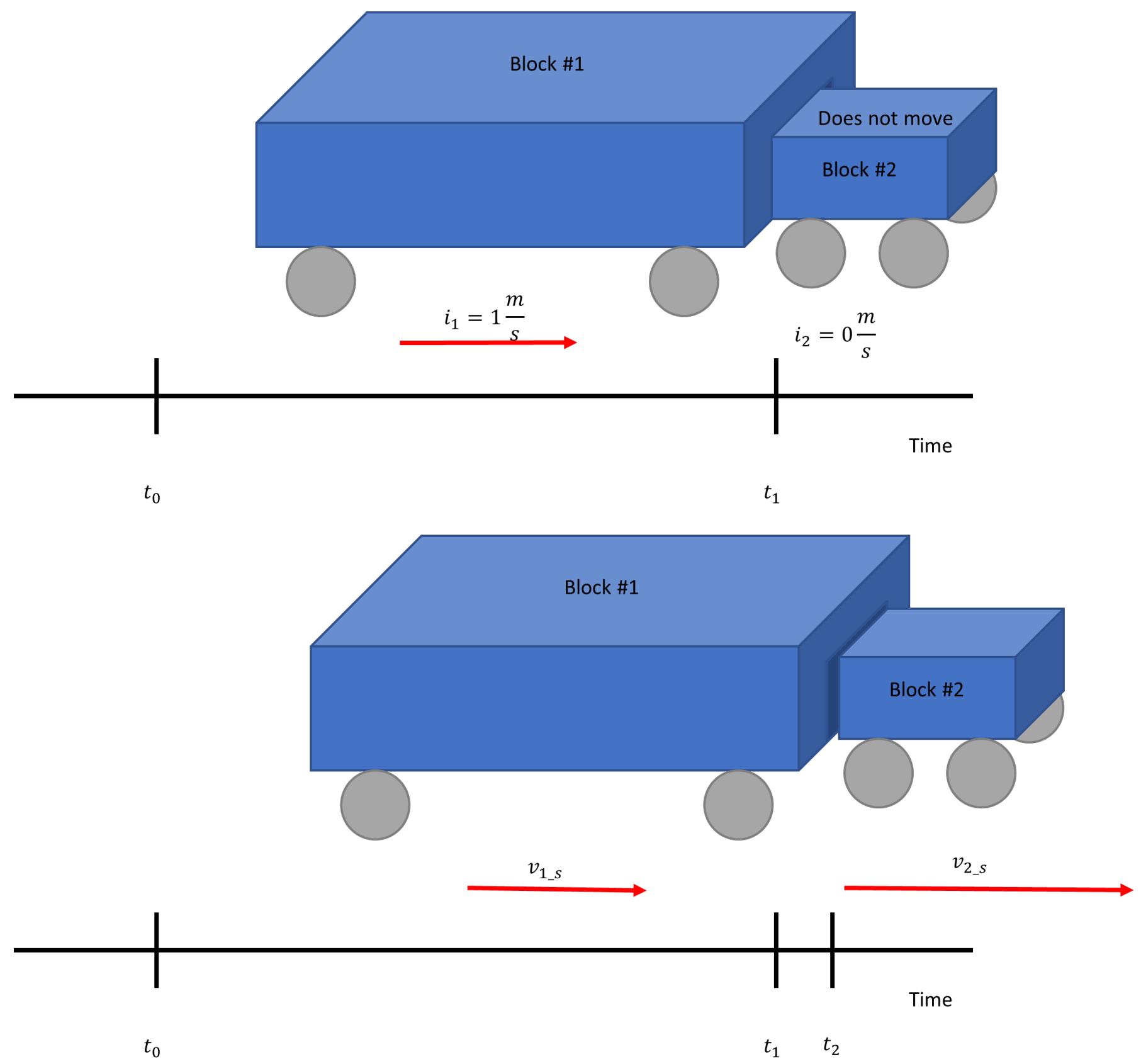

In other words, Block \#2 gains its final speed when the physical contact (impact) between Block \#2 and Block \#1 ends. During the impact, some of the initial kinetic energy $\left(\boldsymbol{K E}_{\mathbf{1}}\right)$ carried by Block \#1 is transferred into heat and shock waves. The shock waves propagate in the steel blocks at speed $5196 \mathrm{~m} / \mathrm{s}$ [20]. The final speeds of Block \#1 and Block \#2 are $\boldsymbol{v}_{\mathbf{1 s}}$ and $\boldsymbol{v}_{\mathbf{2 s}}$ appropriately in according to the formulas (28) and (29).

Finally, consider the inelastic collision in Situation \#2 (with the additional mass). Block \#1 (200kg) collides with Block \#2 $(1 \mathrm{~kg})$. The lengths of and the initial speeds of the blocks do not change. The impact starts at time $\boldsymbol{t}_{\mathbf{1}}$ which is the same as that in Situation \#1 and ends at time $\boldsymbol{t}_{\mathbf{2}}{ }^{\prime}$ which should be more than $\boldsymbol{t}_{\mathbf{2}}$ if the larger deformation happens. During the impact in Situation \#2, some of the initial kinetic energy $\left(\boldsymbol{K} \boldsymbol{E}_{\mathbf{2}}\right)$ carried by the enlarged Block \#1 is transferred into heat and shock waves. Notice that $\boldsymbol{K E}_{\mathbf{2}}>$ $\boldsymbol{K} \boldsymbol{E}_{\mathbf{1}}$ because the total mass of Block \#1 becomes $200 \mathrm{~kg}$ but its initial speed $\mathbf{1}$ meter per second is the 
same as that in Situation \#1. The shock waves propagate in the steel blocks at speed $5196 \mathrm{~m} / \mathrm{s}$. The fields of the particles of Block \#1 start to compress the fields of the particles of Block \#2 at the impact area at the relative speed $\mathbf{1}$ meter per second at time $\boldsymbol{t}_{\mathbf{1}}$. Therefore, the transfer of the kinetic energy from Block \# 1 to Block \#2 runs in the same way as that in Situation \#1. It means that Block \#2 gains enough kinetic energy via the shock wave to begin to move at speed $\boldsymbol{v}_{\mathbf{2 s}}$ at $\boldsymbol{t}_{\mathbf{2}}$. Block \#2 must begin to move at its final speed when its shock wave returns to the impact area at time $\boldsymbol{t}_{\mathbf{2}}$ because a length of Block \#2 and the shock wave speed do not change. However, the impact (with the additional mass) should end at time $\boldsymbol{t}_{\mathbf{2}}{ }^{\prime}$ at the final speeds of the blocks $\boldsymbol{v}_{\mathbf{1}} \mathbf{s}^{\prime}$ and $\boldsymbol{v}_{\mathbf{2} \boldsymbol{s}}{ }^{\prime}$ appropriately if the larger deformation and the impact longer time happen.

Conclusion

The force and the final speed of Block \# 2 increase if a total mass of Block \# 1 increases in according to the formulas (2) and (29) appropriately. Based on the formulas (2) and (3), the elastic deformation should be larger for the inelastic collision with the added mass (Situation \#2) than that for the inelastic collision without the added mass (Situation \#1). It should be so because the force increases. If the initial speeds of and lengths of the blocks do not change, then the only physical way how the larger deformation and the higher final speed of Block \#2 can happen is a longer time of the impact in Situation \#2. However, it contradicts the physics of collision because the shock wave in Block \#2 returns to the impact area at the same time in the inelastic collision with or without the additional mass. Block \#2 must begin its own motion at the same time in the inelastic collision with or without the added mass assembled to Block \#1 if a length of Block \#2 is less than a length of Block \#1. It should be again mentioned that the shock waves in the blocks propagate at the same speed if the blocks are made of the same material.

If the final speed of Block \#2 in the experimental situation \#2 increases but the collision time does not increase, it raises a question how physically the additional mass can make the final speed of Block \#2 higher. The speed increase of Block \#2 may be caused by the kinetic energy transfer. An only known physical way which the initial kinetic energy of Block \#1 can be transferred from Block \#1 to Block \#2 is via the physical interactions of the fields of the particles in the impact area. However, the interactions of the fields begin in the same conditions (initial speeds of the fields, shock wave speed in steel, lengths of the blocks, impact area) in Situation \#1 and Situation \#2. Under such conditions, Block \#2 should start to move at speed $\boldsymbol{v}_{\mathbf{2 s}}$ at time $\boldsymbol{t}_{\mathbf{2}}$ instead of $\boldsymbol{v}_{\mathbf{2 s}}{ }^{\prime}$ at $\boldsymbol{t}_{\mathbf{2}}{ }^{\prime}$ in Situation \#2. That is, Block \#2 should begin to move at its final speed when the shock wave returns to an impact area. This time should be time $\boldsymbol{t}_{\mathbf{2}}$ because the speed of the shock wave in Block \#2 and a length of Block \#2 do not change. The author assumes that maybe the interactions between the particles of the two blocks could be based on some sort of the quantum entanglement, that is, without any fields, or the kinetic energy transfer happens via some sort of the quantum entanglement anisotropically. Though, such an assumption requires a supplementary experimental research. 
To clearly observe whether or not the deformation happens to be larger after the additional mass is assembled, Block \#1 or Block \#2, which is made of a material with a lower Young's modulus $\boldsymbol{E}$, can be used for the experimental collisions. However, one of the blocks should be made of steel. In this way, the deformation of another block should be observable.

References:

1. Serway A. Raymond, Jr., Jewett W. John (2004) Electric Fields, in PHYSICS for Scientists and Engineers with Modern Physics ( $6^{\text {th }}$ Edition, Volume 2 ) Serway A. Raymond, Jr., W. Jewett W. John, California State Polytechnic University-Pomona, USA

2. Serway A. Raymond, Jr., Jewett W. John (2004) Electric Fields, in PHYSICS for Scientists and Engineers with Modern Physics ( $6^{\text {th }}$ Edition, Volume 2) Serway A. Raymond, Jr., W. Jewett W. John, California State Polytechnic University-Pomona, USA

3. Rudden M.N., Wilson J. (1993) Structure of Solids, in Elements of Solid State Physics (Second Edition), Rudden M.N., Wilson J., University of Northumbria at Newcastle, John Wiley \& Sons Ltd, UK, ISBN 0471929735

4. Khlystov Nikita, Lizardo Daniel, Matsushita Keisuke, Zheng Jennie (2013) Uniaxial Tension and Compression Testing of Materials, 3.032 Lab Report, September

25: http://web.mit.edu/dlizardo/www/UniaxialTestingLabReportV6.pdf

5. Grady Dennis (2017) Shocks and structured waves, in Physics of Shock and Impact (Volume 1), IOP Publishing Ltd: https://doi.org/10.1088/978-0-7503-1254-7ch1

6. Britton W. G. B., Fendley J. J., Michael M.E. (1978) Longitudinal impact of rods: A continuing experiment, The American Journal of Physics, Volume 46 (11) - Nov 1, 1124-1130: https://doi.org/10.1119/1.11153

7. Roura P. (1997) Collision duration in the elastic regime, The Physics Teacher, 35(7), 435436: https://doi.org/10.1119/1.2344749

8. Urone Peter Paul Dr., Hinrichs Roger Dr., Dirks Kim Dr., Sharma Manjula Dr. (2017) Linear Momentum and Collisions, in College Physics Urone Peter Paul Dr., Hinrichs Roger Dr., Dirks Kim Dr., Sharma Manjula Dr., Rice University, OpenStax: https://openstax.org/details/books/College-Physics

9. Urone Peter Paul Dr., Hinrichs Roger Dr., Dirks Kim Dr., Sharma Manjula Dr. (2017) Linear Momentum and Collisions, in College Physics Urone Peter Paul Dr., Hinrichs Roger Dr., Dirks Kim Dr., Sharma Manjula Dr., Rice University, OpenStax: https://openstax.org/details/books/College-Physics

10. Urone Peter Paul Dr., Hinrichs Roger Dr., Dirks Kim Dr., Sharma Manjula Dr. (2017) Dynamics: Force and Newton's Laws of Motion, in College Physics Urone Peter Paul Dr., Hinrichs Roger Dr., Dirks Kim Dr., Sharma Manjula Dr., Rice University, OpenStax: https://openstax.org/details/books/College-Physics 11. Khlystov Nikita, Lizardo Daniel, Matsushita Keisuke, Zheng Jennie (2013) Uniaxial Tension and Compression Testing of Materials, 3.032 Lab Report, September 25: http://web.mit.edu/dlizardo/www/UniaxialTestingLabReportV6.pdf 
12. Brown G. Robert $(1993,2007,2013)$ Systems of Particles, Momentum and Collisions, in Introductory Physics I Elementary Mechanics, Brown G. Robert, Duke University Physics Department, Durham,

NC: http://webhome.phy.duke.edu/ rgb/Class/intro_physics_1.php

13. Urone Peter Paul Dr., Hinrichs Roger Dr., Dirks Kim Dr., Sharma Manjula Dr. (2017) Linear Momentum and Collisions, in College Physics Urone Peter Paul Dr., Hinrichs Roger Dr., Dirks Kim Dr., Sharma Manjula Dr., Rice University, OpenStax: https://openstax.org/details/books/College-Physics

14. Urone Peter Paul Dr., Hinrichs Roger Dr., Dirks Kim Dr., Sharma Manjula Dr. (2017) Linear Momentum and Collisions, in College Physics Urone Peter Paul Dr., Hinrichs Roger Dr., Dirks Kim Dr., Sharma Manjula Dr., Rice University, OpenStax: https://openstax.org/details/books/College-Physics

15. Landau L.D., Lifshitz E. M. (1976) Elastic Collisions, in MECHANICS (Third edition), Landau L. D., Lifshitz E. M., Institute of Physical Problem, USSR Academy of Sciences. Translated from the Russian by J.B. Sykes and J.S. Bell, Reed Educational and Professional Publishing Ltd. (1981), ISBN:

0080210228, https://books.google.com/books?id=e-

xASAehg1sC\&dq=isbn\%3A0080210228\&source=gbs_book_other_versions

16. Jackson L. Robert, Green Itzhak, Marghitu B. Dan (2010) Predicting the coefficient of restitution of impacting elastic-perfectly plastic spheres, Nonlinear Dynamics, Volume 60, Issue 3, pp. 217-

229, https://doi.org/10.1007/s11071-009-9591-z

17. Ferreira Da Silva F. M. (2007) Meaning and usefulness of the coefficient of restitution, European Journal of Physics, 28(6): 14 - Nov 1, https://doi.org/10.1088/0143-0807/28/6/019

18. https://en.wikipedia.org/wiki/Coefficient_of restitution

19. Ferreira Da Silva F. M. (2007) Meaning and usefulness of the coefficient of restitution, European Journal of Physics, 28(6): 14 - Nov 1, https://doi.org/10.1088/0143-0807/28/6/019

20. Britton W. G. B., Fendley J. J., Michael M.E. (1978) Longitudinal impact of rods: A continuing experiment, The American Journal of Physics, Volume 46 (11) - Nov 1, 1124-1130: https://doi.org/10.1119/1.11153 